\title{
HarmoSim: A Tool for Harmonic Distortion Simulation and Assessment of Nonlinear Loads
}

\author{
J. BAPTISTA, ${ }^{1}$ R. MORAIS, ${ }^{2}$ A. VALENTE, ${ }^{3}$ S. SOARES, ${ }^{3}$ M. CANDEIAS, ${ }^{1}$ M.J.C.S. REIS ${ }^{3}$ \\ ${ }^{1}$ Department of Engineering, University of Trás-os-Montes e Alto Douro, P-5001-801 Vila Real, Portugal \\ ${ }^{2}$ Department of Engineering/CITAB, University of Trás-os-Montes e Alto Douro, P-5001-801 Vila Real, Portugal \\ ${ }^{3}$ Department of Engineering/IEETA, University of Trás-os-Montes e Alto Douro, P-5001-801 Vila Real, Portugal
}

Received 7 April 2009; accepted 18 May 2011

\begin{abstract}
Electrical power quality (PQ) is a crucial competitive and developing factor to all economic activities. The economic impact resulting from a bad $\mathrm{PQ}$ would be drastic on all consumers. Computers, uninterruptible and switched power supplies (UPS), and fluorescent lamps/tubes are examples of nonlinear loads that have the consumption of a nonsinusoidal current, which cause disturbances in the power supply system (that may be severe or not). This study discusses residential generic power circuitry analysis and simulation, under nonlinear loads, in connection with undergraduate electrical engineering education. It briefly reviews some of the basic techniques, and presents a software tool that has been found to be very useful in the context. The tool has an easy-to-use, friendly interface, and can be used to teach design techniques or as a laboratory support to study the applicability of known methods to real situations. The students can perform simulations with their own data on Microsoft ${ }^{\mathrm{TM}}$ Windows ${ }^{\mathbb{R}}$-based platforms. (c) 2011 Wiley Periodicals, Inc. Comput Appl Eng Educ 22:340-348, 2014; View this article online at wileyonlinelibrary.com/journal/cae; DOI $10.1002 /$ cae.20559
\end{abstract}

Keywords: residential power circuits; simulation; harmonic distortion; nonlinear loads

\section{INTRODUCTION}

Electric power generation, transportation, and consumption are among the problems often faced by anyone working in the broad field of Electrical Engineering. The problem of understanding and teaching harmonics mechanisms and its implications has attracted the scientific community for decades, and many are the works published in the field [1-5], to name only a few. For this reason, an introductory course focusing on electrical energy systems would be welcome in addition to the background of Electrical Engineering students, especially to those interested in electrical power generation, transportation, consumption, or electrical systems in general.

Solving problems while experimenting and comparing the available algorithms is of paramount importance in the learning process. In this study, we present a computer application

Correspondence to M.J.C.S. Reis (mcabral@utad.pt).

() 2011 Wiley Periodicals Inc. intended to be an easy-to-use tool to help the students do that, and which is being used by Electrical Engineering students of the Electrical Engineering Department of the University of Trás-os-Montes e Alto Douro (http://www.utad.pt/en/teaching/ degrees/ents/electronics/index.html). The tool allows the students to try several loads on their own data, and then compare the results (and consequently infer the behavior of the loads) in an easy-to-use environment that is similar to a laboratory. The computer application was named "HarmoSim," standing for "Harmonic Simulation." The main function of this tool was the simulation of individual (isolated) electric loads and residential power circuits with several kinds of different loads. It enables the graphical visualization of different parameters related to circuit electric quality; among them are the total harmonic distortion (THD), crest factor, power factor, and line current. The simulated signals for the harmonic spectrum and current and voltage waves are also plotted.

We believe that a small introduction to electrical energy quality, nonlinear loads, and harmonic distortion parameters will help to clarify the ideas that lead to the tool development. The following sections are dedicated to this. 


\section{BACKGROUND}

Electrical power is usually distributed in sinusoidal three-phase power lines. Parameters such as frequency, amplitude, shape, and symmetry are usually used to characterize the voltage and current waves and its purity degree, that is, how near or far it is from a perfect sinusoidal waveform [6]. However, the transportation and distribution processes, as well as energy consumption itself, introduce distortions or disturbances, and parameters' variation, affecting the quality of the energy supplied to all consumers.

\section{Electrical Power Quality and Nonlinear Loads}

The ongoing technological development demands for more efficient and better quality in the electrical energy supply. It is being viewed as an important economic competitive factor to the industry, and services in general, particularly during the last few decades. A great concern is being noticed, mainly in the industry, in order to minimize the economic risks resulting from a poor or bad power quality (PQ).

There are a great number of equipments very sensitive to disturbances occurring at the line supply, particularly electronic equipments like computers, TV sets, among others. A huge effort is also being made in order to define criteria to evaluate $\mathrm{PQ}$, and relating these criteria to the equipments' disturbances admissible functioning limits [7,8]. In addition, there are a number of international standards limiting electric disturbances $[9,10]$.

A load is said to be nonlinear when it has different waveforms for the consumed current and the supplied voltage. Examples of nonlinear loads are uninterruptible and switched power supplies (UPS), compact and traditional fluorescent lamps/tubes, controlled rectifiers, and TV sets. Figure 1 shows the current and voltage typical consumption waveforms for a personal computer (PC).

\section{Harmonic Distortion Indicators}

There are a number of different ways to characterize and describe a periodic signal. Here we are interested in a set of parameters that may be used to measure and characterize the signals' harmonic distortion. The Fourier series is the tool used for analyzing the harmonic contents of a signal. Any periodic signal may be represented by its Fourier series [11], as

$$
y(t)=H_{0}+\sum_{n=1}^{\infty} H_{n}^{2} \sqrt{2} \sin \left(n \omega t+\varphi_{n}\right)
$$

where $H_{n}$ represents the $n$th harmonic. $H_{0}$ corresponds to the DC component (which is null to odd signals and half wave symmetric signals). The advantages and limitations of Fourierbased analysis methods are widely documented in the literature in its various flavors and applications [12-16].

From the set of techniques used for evaluating the voltage and current harmonic distortion the current root-mean-squared (RMS) value, power factor, crest factor, distortion power, frequency spectrum, and harmonic distortion are among the most usefully used. We must know/understand these values in order to introduce possible correction actions.

The current RMS value can be defined by

$$
I_{\mathrm{RMS}}=\sqrt{\sum_{n=1}^{\infty} H_{n}^{2}}
$$

which can be rewritten as

$$
I_{\mathrm{RMS}}=\sqrt{I_{1}^{2}+I_{3}^{2}+I_{5}^{2}+\ldots+I_{n}^{2}}
$$

where $I_{n}$ represents the current associated with the $n$th odd harmonic. The greater the $n$ is the lower the influence on the $I_{\text {RMS }}$ value. Usually the first harmonics will suffice to have a good approximation to the correct value. This value is also connected to the Joule's thermal effect.

The THD rate was introduced as a need to numerically quantify the current or voltage harmonics existing in a specific electric system spot. According to IEEE 519-1992 recommended practices and requirements for harmonic control in electrical power systems [9], THD is defined as the ratio between the harmonics' RMS value and the fundamental component, that is,

$$
\operatorname{THD}(\%)=\frac{\sqrt{\sum_{n=2}^{\infty} H_{n}^{2}}}{H_{1}} \times 100
$$

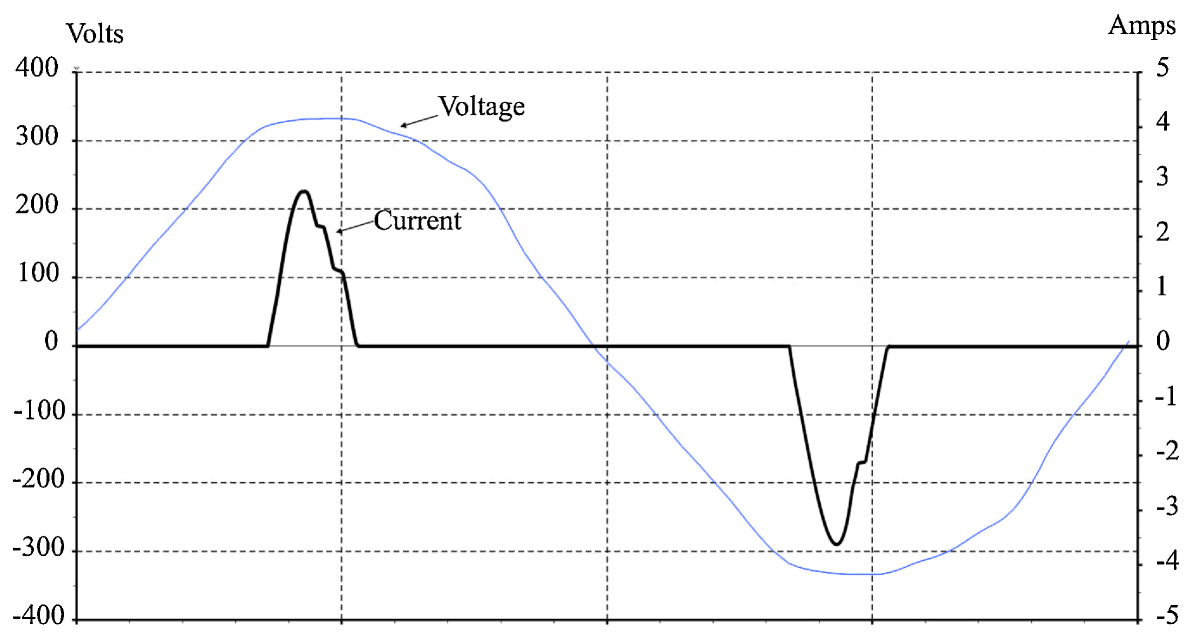

Figure 1 Current and voltage typical consumption wave forms for a personal computer. [Color figure can be viewed in the online issue, which is available at wileyonlinelibrary.com.] 
When no distortion is present $\mathrm{THD}=0$; this means that a null or low THD is the goal to achieve.

The power factor defined by

$$
\mathrm{PF}=\frac{\cos \varphi}{{\sqrt{1+\mathrm{THD}_{i}^{2}}}^{2}}
$$

where $\varphi$ represents the phase-shift between the fundamental current and voltage components, is another parameter deeply affected by the introduction of harmonic distortion in the current: the far from one this parameter is the greater the current distortion.

By definition, the crest factor, also called "peak-to-RMSratio" or "peak-to-average-ratio," corresponds to the ratio between the peak of the current or voltage value and its RMS value,

$$
C_{V}=\frac{V_{\mathrm{max}}}{V_{\mathrm{RMS}}}, \quad C_{I}=\frac{I_{\max }}{I_{\mathrm{RMS}}}
$$

If the signal is a perfect sinusoid then the crest factor value will be equal to $\sqrt{2}$. If the crest factor value is different from $\sqrt{2}$ we have a distorted signal. Nonlinear loads typically have current crest factors above $1.5(\sqrt{2} \approx 1.414)$, reaching 5 in some critical situations. The current value can be 1.5-5 times the RMS value, which affects the ability of some circuits to deal with these surges.

\section{Modeling}

In order to evaluate and understand the kind of disturbances that are usually injected in residential power circuitry during normal equipment operation/utilization, we have prepared a set of monitoring tests. During these tests we have measured the different parameters for different loads and functioning limits, using a PP-4300 Dranetz-BMI analyzer, (www.dranetz-bmi.com), and the data were treated in the DranView software package (www.dranetz-bmi.com). We have concluded that there are harmonic patterns repeating themselves for each kind of load, both for current and voltage waveforms. Figure 2 (voltage (a) and current (b)) shows the results for 58W fluorescent tubes (OSRAM L 58W/765). For each current and voltage harmonic, we have calculated the mean of each component value from all tests. The amplitude and phase odd harmonics were then inserted in the tool's database.

From a harmonic point of view, the electric loads are the ones creating more uncertainty, because we do not know its nature (resistive, mainly inductive, or capacitive) as well as its stationary functioning regimen. The equivalent circuit of a nonlinear load will always be a series and/or parallel of capacitors, resistors, and inductors. The common approach to characterize a certain nonlinear load is to measure its harmonic voltage supply, $V(n)$, and the current it absorbs, $I(n)$, being its impedance given by

$$
Z(n)=\frac{V(n)}{I(n)}
$$

The standard harmonic pattern of all loads used during the development of the tool presented here was found using this methodology.

An electrical installation may be approximated by the equivalent electric circuit presented in Figure 3, where each parallel impedance represents a load linked to the supply feeder. Therefore, we may simulate and analyze the global behavior of an electrical circuit with nonlinear loads.

The total input current $I_{i}(n)$ will be equal to

$$
I_{i}(n)=I_{1}(n)+I_{2}(n)+I_{3}(n)+\ldots+I_{k}(n)
$$

where $n$ represents the $n$th harmonic and $k$ the number of simultaneously connected loads.

To find the total voltage $V_{i}(n)$ it is necessary to determine the equivalent impedance, as shown in Figure 4. Because all loads are parallely connected, the total impedance load will be given by

$$
Z_{\mathrm{eq}}(n)=\frac{1}{Y_{\mathrm{eq}}(n)}
$$

where

$$
Y_{\text {eq }}(n)=Y_{1}(n)+Y_{2}(n)+Y_{3}(n)+\ldots+Y_{k}(n)
$$

is the total admittance.

The harmonic voltage will then be given by the Ohm's law,

$$
V_{i}(n)=Z_{\mathrm{eq}}(n) \times I_{i}(n)
$$

We are now able to estimate the current RMS value (Eq. 2), THD value (Eq. 3), power factor value (Eq. 4), crest factor value (Eq. 5), the spectral harmonics (frequency), and the voltage and current (time) wave forms (recovered from the Fourier series, defined by Eq. 1).

\section{THE TOOL AND SOME SIMULATION RESULTS}

The HarmoSim tool has been developed under the Visual Basic.NET programming environment. The tool has five main blocks/utilities: loads management, loads analysis, circuitry simulation, reports history, and help.

The loads management block is used to manage and characterize all loads existing in the database (or to be inserted). The first 25 odd harmonics, phases, nominal current, and power voltage (according to measures) must be imputed. (The even harmonics are not used because, theoretically, they are null when the signal under analysis, $f(t)$, is odd, that is, when $f(t)=-f(-t)$. In our case, we are not working with perfectly odd signals, but the values of the even harmonics are very much smaller than the values of the odd harmonics.) All these parameters associated with a particular load can be edited and/ or deleted.

There are several characteristics associated with the loads analysis that can be viewed, edited, or deleted in the corresponding block. This includes the harmonic spectrum, and current and voltage waveforms (both time domain and frequency domain). A Visual Basic.NET "ListView" is used to show the harmonics, current, and voltage in absolute value or as a percentage of the RMS value; the voltage and current spectrums plots; the time domain current and voltage plots; and the power factor and crest factor (both current and voltage). The THD, both for current and voltage, are calculated and presented according to Refs. [9] and [10] standards. A PDF report is generated, under user demand, which includes all the parameters and plots associated with the selected load. 


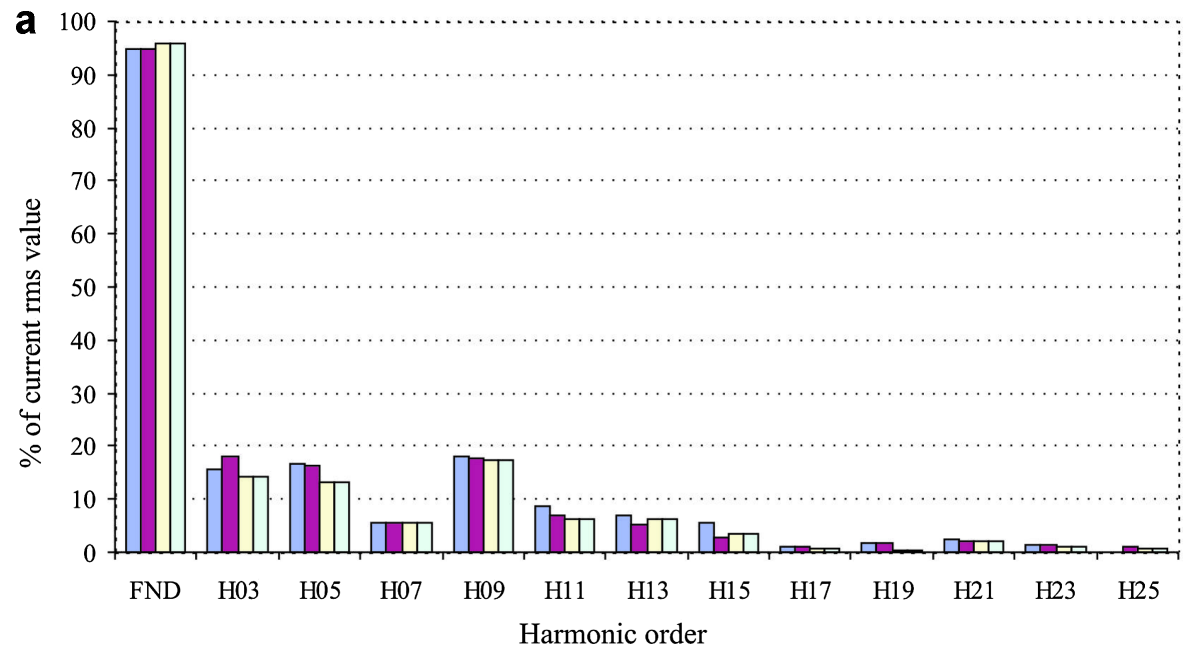

$\square$ Fluorescent $1 \square$ Fluorescent $2 \square$ Fluorescent $3 \square$ Fluorescent 4

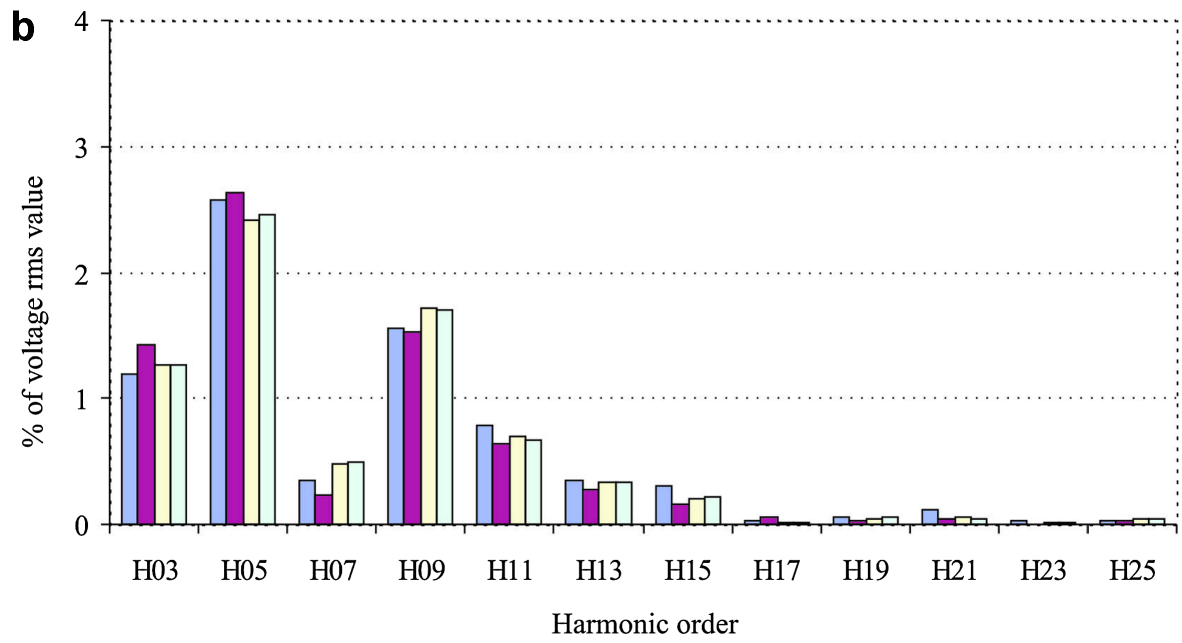

$\square$ Fluorescent $1 \square$ Fluorescent $2 \square$ Fluorescent $3 \square$ Fluorescent 4

Figure 2 Measured harmonic spectrums of 58W fluorescent tubes (OSRAM L 58W/765): (a) current, (b) voltage. [Color figure can be viewed in the online issue, which is available at wileyonlinelibrary.com.]

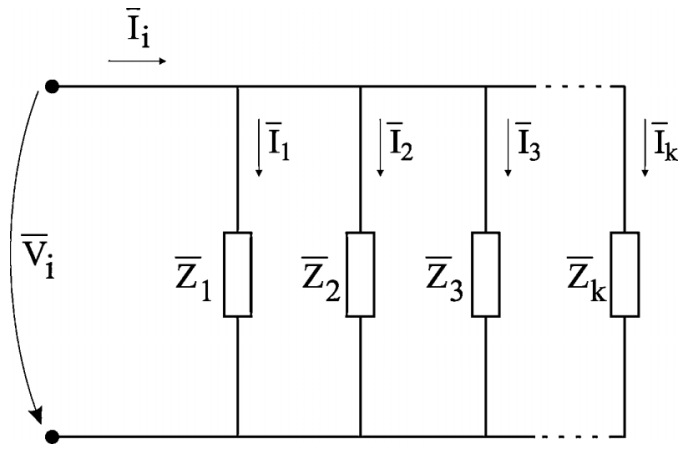

Figure 3 Equivalent circuit of an electrical installation.

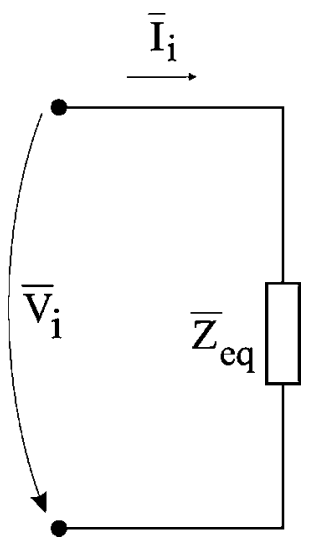

Figure 4 Electrical installation simplified model (total equivalent impedance). 


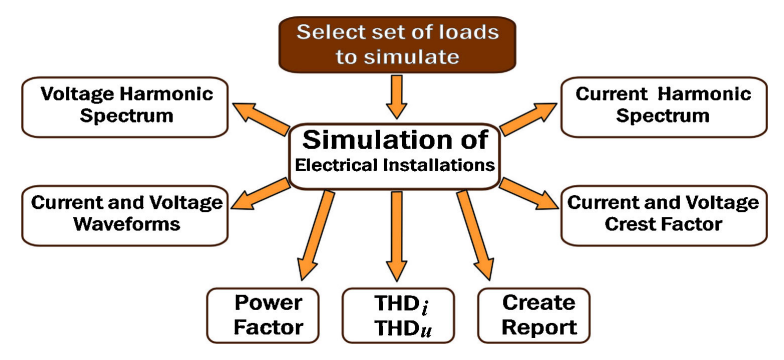

Figure 5 Circuitry simulation block diagram. [Color figure can be viewed in the online issue, which is available at wileyonlinelibrary. com.]

The circuitry simulation block is where actually all the simulations and behavior preview is done for a single- or threephase set of loads, which the user must choose. Figure 5 shows its block diagram. The user's first step must be the choice of loads to simulate from those existing in the database; the different kind, number of loads and phase must be inserted. Next, the user chooses the phase to simulate (R, S, or T), because only one phase at a time can be simulated. The different plots and parameters are then shown, including the current and voltage spectrum harmonics, THD, circuit power factor, and crest factors. Figure 6 shows the HarmoSim tool circuitry simulation window.
The reports history block may be used to see all the previously saved reports and look at a particular simulation. The help block explains, in a summarized way, the tool's functioning principles.

In order to compare the results of the simulations with the real values (measured using the Dranetz-BMI analyzer), we have prepared a set of monitoring tasks, using different loads in different operating conditions, which were implemented by our students. These monitoring tasks were conducted in the campus of the University of Trás-os-Montes e Alto Douro, houses and flats, and small industry. Here we present two of such case studies.

Figure 7 shows the current (a) and voltage (b) plots, measured and simulated, for 6 PCs and 19, 58W, fluorescent lamps without capacitive compensation (OSRAM L 58W/765). Table 1 shows the corresponding THD, power factors, crest factors, and total current. Data for the second case study are shown in Figure 8 (current (a) and voltage (b)) and Table 2. This time, $1 \mathrm{PC}, 1 \mathrm{TV}$ set, and 1 laser printer were used.

As can be seen from the examples above, good approximation results are achieved by the simulations produced by the HarmoSim tool. The differences between the real (measured) and simulated signals are listed in Table 3 for the average and $l^{\infty}$ error $\left(\|x\|^{\infty}=\max \left|x_{i}\right|\right)$.

The HarmoSim tool is being successively used to teach the influences of harmonic distortion in the quality of electrical

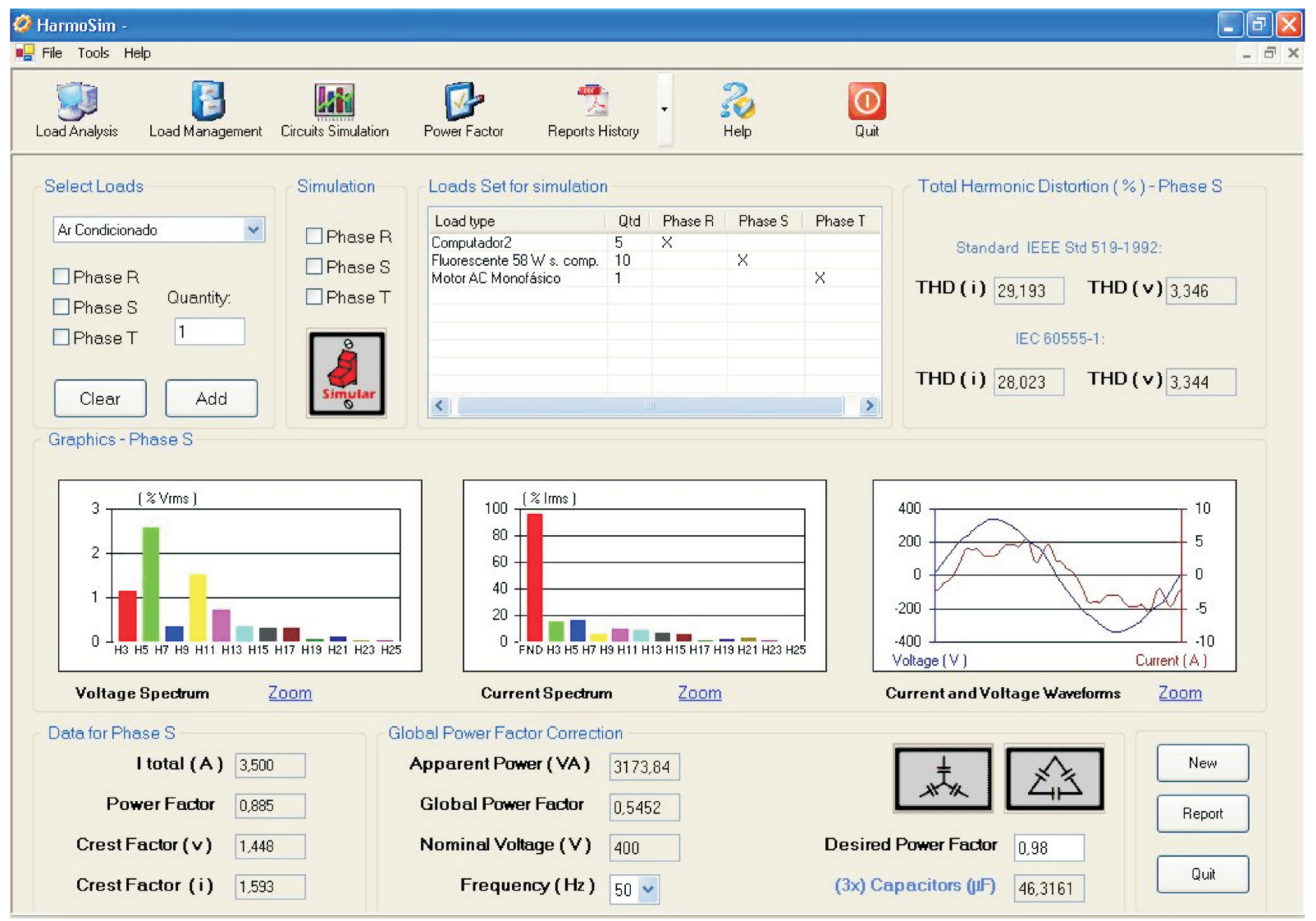

Figure 6 Circuitry simulation block typical window. [Color figure can be viewed in the online issue, which is available at wileyonlinelibrary.com.] 

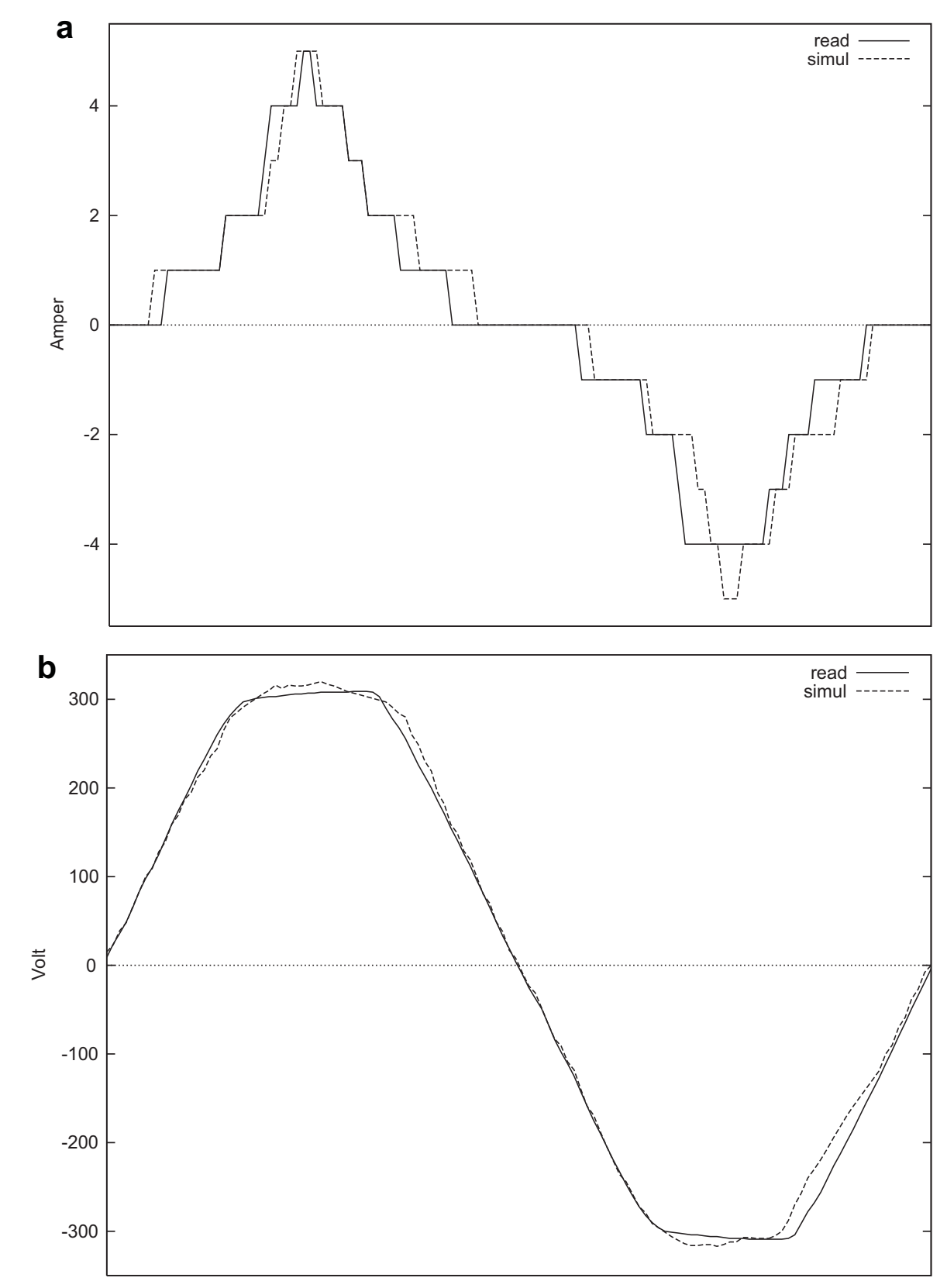

Figure 7 Results for the first case study: 6 personal computers and 19, 58W, fluorescent tubes without compensation (OSRAM L 58W/765): (a) current and (b) voltage.

Table 1 Results for the First Case Study: 6 Personal Computers and $1958 \mathrm{~W}$ Fluorescent Tubes Without Compensation (OSRAM L $58 \mathrm{~W} / 765)$

\begin{tabular}{lcc}
\hline Parameter & Measured & Simulated \\
\hline $\operatorname{THD}_{V}(\%)$ & 4.25 & 3.6 \\
$\operatorname{THD}_{I}(\%)$ & 33.1 & 31.16 \\
$I_{\text {total }}(\mathrm{A})$ & 2.47 & 2.47 \\
Power factor & 0.95 & 0.95 \\
Current crest factor & 1.9 & 2.14 \\
\hline
\end{tabular}

energy supply to the students of Electrical Engineering curriculum at the University of Trás-os-Montes e Alto Douro. It is being used in the Electrical Power Distribution Networks, Energy Management and Quality, and Safety in Electrical Power Distribution Networks courses of that curriculum.

\section{CONCLUSIONS}

Circuitry analysis and simulation tasks are among the problems that an Electrical Engineer often faces. An undergraduate course in the subject not only provides the student with relevant 

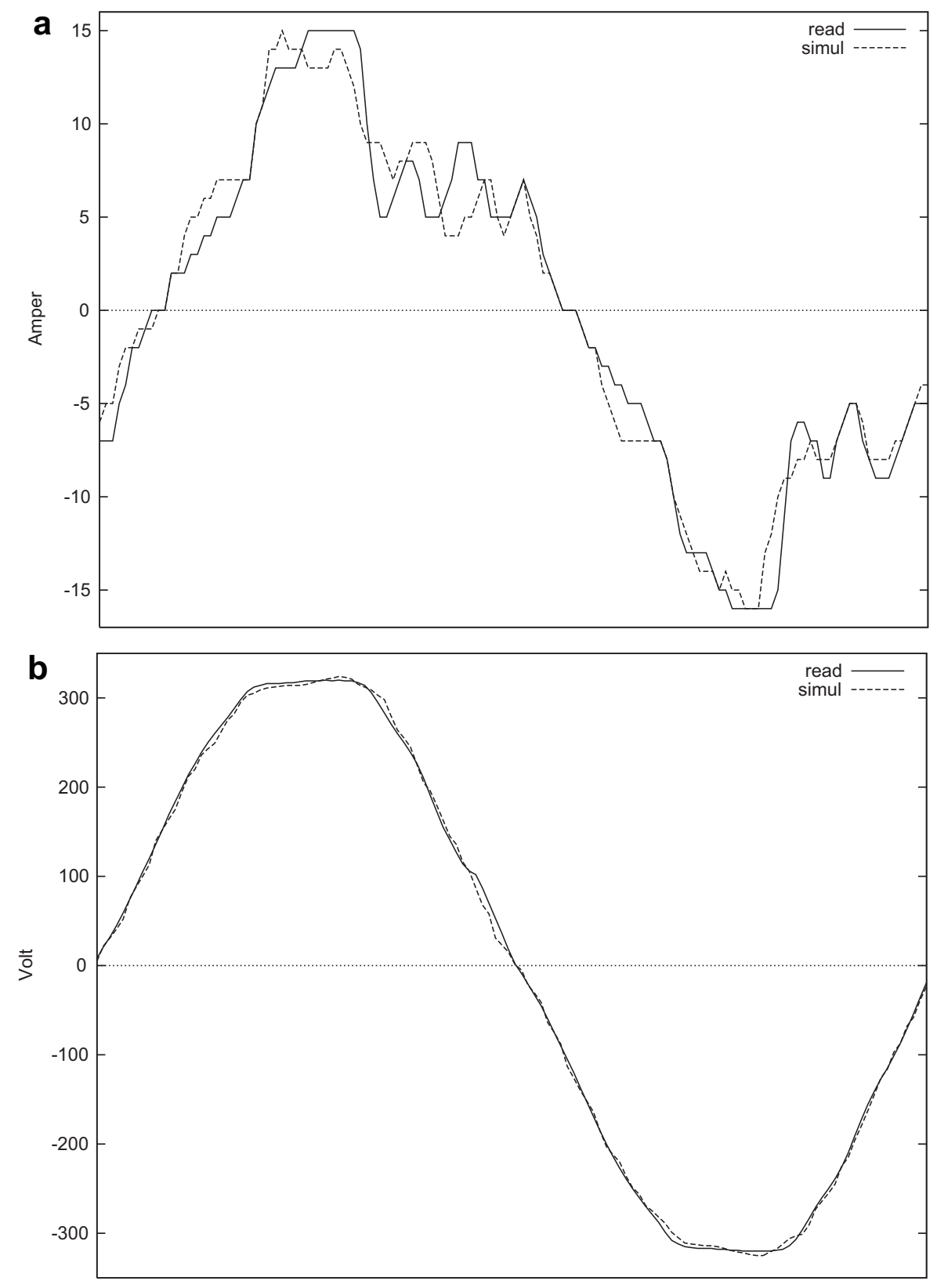

Figure 8 Results for the second case study: 1 personal computer, $1 \mathrm{TV}$, and 1 laser printer: (a) current and (b) voltage.

Table 2 Results for the Second Case Study: 1 Personal Computer, 1 TV, and 1 Laser Printer

\begin{tabular}{lcr}
\hline Parameter & Measured & Simulated \\
\hline $\operatorname{THD}_{V}(\%)$ & 2.45 & 2.51 \\
$\operatorname{THD}_{I}(\%)$ & 17.49 & 17.48 \\
$I_{\text {total }}(\mathrm{A})$ & 7.87 & 7.92 \\
Power factor & 0.78 & 0.78 \\
Current crest factor & 1.51 & 1.53 \\
\hline
\end{tabular}

Table 3 Average and $1^{\infty}$ Error Results for the Two Case Studies Presented

\begin{tabular}{lcr}
\hline & \multicolumn{2}{c}{ Error } \\
\cline { 2 - 3 } Case study & Average & $l^{\infty}$ \\
\hline 1-Voltage & 4.5 & 22.2 \\
1-Current & 1.3 & 5.3 \\
2-Voltage & 8.5 & 38.5 \\
2-Current & 0.4 & 1.7 \\
\hline
\end{tabular}


expertise concerning the problems, but may also be of considerable value in understanding how a background in Fourier and numerical analysis can be applied in the field to concrete simulation problems.

In this context, we have experienced the need for tools that could be easily used inside or outside the classroom, and at the same time were easy to update and maintain. The tool that we have described allows the student to try different scenarios on their own data (different number and types of electrical devices), and then compare the results in an easy-to-use environment that is similar to a laboratory, in a computer system using Microsoft ${ }^{\mathrm{TM}}$ Windows ${ }^{\mathrm{R}}$.

The tool can be used by the students in their daily work, inside or outside the classroom, and, in our view, has proved to be a valuable tool for teaching and understanding electric circuitry analysis and simulation, especially the ones presented above.

For certain applications, such as static AC/DC power converters (of 6 and 12 pulses) and cycle converters usually used in $\mathrm{AC}$ motors control, more than 25 odd harmonics will typically be needed in order to produce a good approximation of the Fourier series to the signal. The authors are currently working on a new software tool version in order to include a greater number of harmonics $(>25)$ and all the three phases during the simulation process at once.

\section{REFERENCES}

[1] J. Schlabbach, Practical approach to teach harmonics-simulation, measurement and assessment, In Proceedings of the 12th IEEE Mediterranean Electrotechnical Conference, MELECON 2004, Vol. 3, pp 1153-1156.

[2] R. A. Rob and W. T. Jewell, Computer based harmonic simulation and testing for microprocessor-based phase distance relay with phase locator, In Proceedings of the Industrial and Commercial

\section{BIOGRAPHIES}

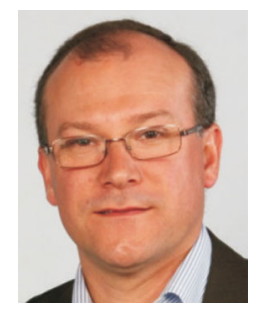

José Baptista graduated in Electrical Engineering from the University of Trás-os-Montes e Alto Douro (UTAD), Portugal in 1991. He obtained the M.Sc. degree in Power Electronics in 1997 from UTAD and the Ph.D. degree in Electrical Engineering in 2007 from UTAD. Presently, he is an Assistant Professor in the Department of Electrical Engineering, UTAD. $\mathrm{He}$ is also a researcher in power quality, electrical machines and renewables. His maininterest areas are power quality, electrical machines and renewables.

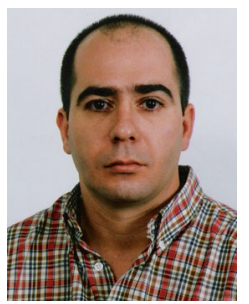

Raul Morais graduated in Electrical Engineering from UTAD, Portugal in 1993. He obtained the M.Sc. degree in Industrial Electronics in1998 from the University of Minho, Portugal and the Ph.D. degree in Microelectronics in 2004 from the UTAD. Presently, he is an Assistant Professor in the Department of Electrical Engineering, UTAD. He is also a researcher in the Signal Processing and Biotelemetry group and the Center for the Research and Technology of Agro-Environment and Biological Sciences (CITAB), and he is involved in the development of instrumentation solutions, energy harvesting technologies and mixed-signal sensing interfaces for agricultural applications. He is also a research collaborator at the Institute of Electronics and Telematics Engineering of Aveiro (IEETA).
Power Systems Technical Conference, 1993, pp. 70-77, May 1993.

[3] P. Lok-Fu, V. Dinavahi, G. Chang, M. Steurer, and P. F. Ribeiro, Real-time digital time-varying harmonic modeling and simulation techniques-IEEE task force on harmonics modeling and simulation, IEEE Trans Power Deliv 22 (2007), 1218-1227.

[4] I. Colak, S. Demirbas, S. Sagiroglu, and E. Irmak, A novel webbased laboratory for DC motor experiments, Comput Appl Eng Educ 19 (2011), 125-135.

[5] S. Tuncer, A new integrated education environment for induction motor drives, Comput Appl Eng Educ 19 (2009). Published online in Wiley Online Library.

[6] J. Arrillaga and N. R. Watson, Power system harmonics, John Wiley and Sons, London, UK, 1985.

[7] R. C. Dugan, M. F. McGranaghan, and H. W. Beaty, Electrical power systems quality, McGraw-Hill, New York, 1996.

[8] S. Santoso, Time-domain power system simulator as an efficient tool for teaching and learning electric power quality phenomena, Comput Appl Eng Educ 17 (2009), 214-224.

[9] IEEE 519-1992, Recommended practices and requirements for harmonic control in electrical power systems, 1992.

[10] IEC 60555-1, Disturbances in supply systems caused by household appliances and similar electrical equipment—Parte 1: Definitions, 1997.

[11] E. O. Brigham, The fast Fourier transform, Prentice-Hall, Englewood Cliffs, NJ, 1974.

[12] J. W. Cooley, How the FFT gained acceptance, IEEE Signal Process Mag 9 (1992), 10-13.

[13] E. B. van Vleck, The influence of Fourier series upon the development of mathematics, Science 39 (1914), 113-124.

[14] A. Papoulis, Signal analysis, McGraw-Hill Book Company, USA, 1977.

[15] T. Blu and M. Unser, Quantitative Fourier analysis of approximation techniques: Part I-interpolators and projectors, IEEE Trans Signal Proces 47 (1999), 2783-2795.

[16] G. Strang and G. Fix, A Fourier analysis of the nite element variational method. In: Constructive aspects of functional analysis, Cremonese, Italy, 1971, pp 796-830.

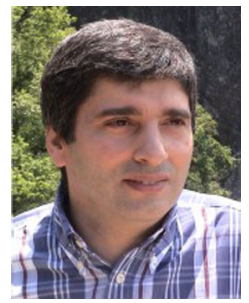

António Valente graduated in Electrical Engineering from UTAD, Portugal in 1994. He obtained the MSc degree in Industrial Electronics in 1999 from the University of Minho, Portugal and the $\mathrm{PhD}$ degree in Microelectronics in 2004 from UTAD. Presently, he is an Assistant Professor in the Engineering Department, UTAD. He is also a researcher at IEETA and he is involved in the research of silicon microsensors.

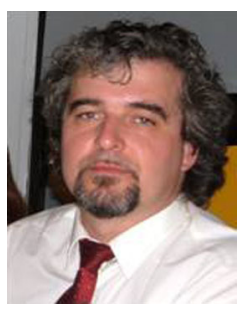

Salviano Soares graduated in Electrical Engineering from UTAD, Portugal in 1991. He obtained the M.Sc. degree in Electronics and Telecommunications in 1995 from University of Aveiro (UA), Portugal, and the Ph.D. degree in Electrical Engineering in 2005 from UA. Presently, he is an Assistant Professor at the Department of Electrical Engineering, UTAD, and also a researcher at IEETA. His main interest area is Digital Signal Processing. 


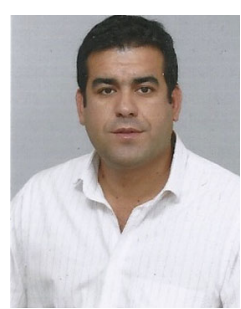

Miguel Candeias graduated in Electrical Engineering from UTAD, Portugal in 2006. $\mathrm{He}$ is a teacher at the "Escola Secundária Morgado de Mateus" school, Vila Real, Portugal. Presently, he is pursuing a M.Sc. degree in Electrical and Electronic Engineering, from the UTAD. His research interests are in the field of e-learning. He is also promoting technology adoption for improving several student activities inside school, such as tagging and interaction using smartphones.

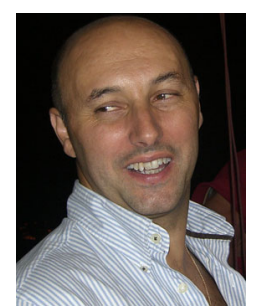

M.J.C.S. Reis received the $\mathrm{PhD}$ degree in Electrical Engineering and the MSc degree in Electronics and Telecommunications from the University of Aveiro, Portugal. Currently he is Associate Professor (Agregado) in the Engineering Department, UTAD, Portugal. He is also a researcher at IEETA. He is the director of the Signal Processing and Biotelemetry research group of UTAD. His research interests are in the area of signal processing, and include modelling and approximation, and problems such as sampling, interpolation, and signal reconstruction. Since 2000 he was co-responsible for eight research projects related to Information Technologies and Internet dissemination and use as a learning/teaching tool all over the Trás-os-Montes e Alto Douroregion. 